\title{
Significant Figures and False Precision
}

Published online: 1 August 2018

(C) ASM International 2018

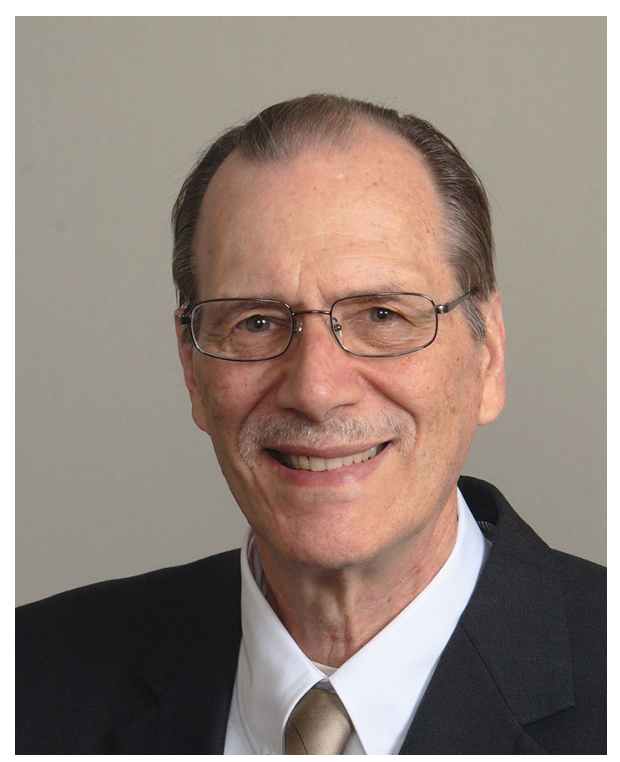

John Morral

A story exists of a museum guide who explains to visitors that several bones on display are 160 million and 5 years old. When questioned about the five extra years, the guide replies, "5 years ago an archeologist told me that the bones were 160 million years old." Some might view this as an amusing story, but for scientists and engineers it is a cautionary tale about significant figures and false precision.

Significant figures are an important scientific concept in which it is assumed that all significant figures in a number are accurate except for the final digit. The final digit is uncertain either because the number was rounded off or because numbers in experiments or calculations were found to vary.
The numbers 430, 43, 4.3, 0.43 and 0.043 all have two significant figures. The location of decimal points is not a factor. The uncertainty of 43 depends on how it was determined. If it was rounded off, the number is between 42.5 and 43.5 and is written as $43 \pm 0.5$ with 0.5 being the uncertainty. The uncertainty of other numbers depends on their source. In scientific work, numbers are often the result of averages obtained from multiple experiments. Then the uncertainty is given by the standard deviation rounded off to one digit to obtain the number of significant figures.

When the museum guide gave the age of the bones as $160,000,005$ years old, the age became a number with nine significant figures. That is obviously wrong and is an example of false precision. Wiki writes, "False precision (also called overprecision, fake precision, misplaced precision and spurious precision) occurs when numerical data are presented in a manner that implies better precision than is justified."

I am concerned when seeing manuscripts written with standard deviations having two or more significant figures. As shown in the following example, uncertainties with two or more significant figures add additional digits to the average. The additional digits are uncertain and a result of false precision.

\begin{tabular}{lll}
\hline & Average & \multicolumn{1}{c}{ SD } \\
\hline Experimental numbers & 7.31732 & 0.382521 \\
Significant figures with 1-digit uncertainty & 7.3 & 0.4 \\
$\begin{array}{l}\text { False significant figures with 2-digit } \\
\quad \text { uncertainty }\end{array}$ & 7.32 & 0.38 \\
$\begin{array}{l}\text { False significant figures with 3-digit } \\
\quad \text { uncertainty }\end{array}$ & 7.317 & 0.383 \\
\hline
\end{tabular}


The first line in the example gives the average of raw data obtained from repeated measurements, along with the standard deviation of the average. The second line illustrates how rounding off the standard deviation to one digit determines the number of significant figures in the average value. The last two lines appear to be more accurate, because of their additional digits, but they are a problem. In addition to giving readers a false sense of accuracy, additional digits slow, and possibly prevent, proper interpretation of text and tables by making them unnecessarily complex.
This editorial contains little new information. It is intended to be a reminder that significant figures are extremely important. Whether writing or reviewing a paper, the accuracy and uncertainty of numbers should be a foremost consideration.

\author{
John Morral \\ Editor-in-Chief \\ Journal of Phase Equilibria and Diffusion
}

